\title{
3D spectroscopy of Wolf-Rayet HII galaxies
}

\author{
C. Kehrig ${ }^{1}$, E. Pérez-Montero' ${ }^{1}$, J. M. Vílchez ${ }^{1}$, J. Brinchmann ${ }^{2}$, \\ D. Kunth ${ }^{3}$, F. Durret ${ }^{3}$, J. Iglesias-Páramo ${ }^{1}$ and \\ J. Hernández-Fernández ${ }^{4}$ \\ ${ }^{1}$ Instituto de Astrofísica de Andalucía - Apartado de correos 3004, 18080 Granada, Spain \\ ${ }^{2}$ Leiden University - PO Box 9513, 2300 RA Leiden, The Netherlands \\ ${ }^{3}$ Institut d'Astrophysique de Paris - 98 bis boulevard Arago, 75014 Paris, France \\ ${ }^{4}$ Universidade de São Paulo (IAG) - Rua do Matão 1226, 05508-090 SP, Brazil
}

\begin{abstract}
Wolf-Rayet HII galaxies are local metal-poor star-forming galaxies, observed when the most massive stars are evolving from O stars to WR stars, making them template systems to study distant starbursts. We have been performing a program to investigate the interplay between massive stars and gas in WR HII galaxies using IFS. Here, we highlight some results from the first 3D spectroscopic study of Mrk 178, the closest metal-poor WR HII galaxy, focusing on the origin of the nebular HeII emission and the aperture effects on the detection of WR features.
\end{abstract}

Keywords. galaxies: dwarf — ISM: abundances — ISM: HII regions — stars: Wolf-Rayet

\section{Introduction}

HII galaxies are local, dwarf starburst systems (e.g., Kehrig, Telles, \& Cuisinier 2004; Westera et al. 2004), which show low metallicity $\left[1 / 50 \lesssim \mathrm{Z} / \mathrm{Z}_{\odot} \lesssim 1 / 3\right]$ (e.g., Kehrig et al. 2006; Pérez-Montero et al. 2009; Cairós et al. 2009, 2010). Wolf-Rayet (WR) signatures (commonly a broad feature at $\sim 4680 \AA$ or blue bump), indicating the presence of WR stars, have been found in the spectra of some HII galaxies (e.g., Kunth \& Sargent 1981; Kehrig et al. 2013). This is an important observational fact since according to recent stellar evolution models for single rotating/non-rotating massive stars, hardly any WRs are expected in metal-poor environments (Leitherer et al. 2014). Studying the WR content in HII galaxies is crucial to test stellar evolutionary models at low metallicities. We have initiated a program to investigate HII galaxies with WR features using integral field spectroscopy (IFS; e.g., Kehrig et al. 2013; Pérez-Montero et al. 2013). So far, we have observed $15 \mathrm{WR}$ galaxies with the optical IFUs: PMAS at the $3.5 \mathrm{~m}$ telescope at CAHA and INTEGRAL at the $4.2 \mathrm{~m}$ WHT in ORM. IFS has many benefits in a study of this kind, in comparison with long-slit spectroscopy. Using IFS one can locate and find WRs where they were not detected before, not only because it samples a larger area of the galaxy, but also because IFS can increase the contrast of the WR bump emission against the galaxy continuum, thus minimizing the WR bump dilution. Also, IFS is a powerful technique to probe issues related with aperture effects, and allows a more precise spatial correlation between massive stars and nebular properties (e.g., Kehrig et al. 2008, 2013).

\section{Results and Conclusions}

We summarize here some recent results on Mrk178, one of the most metal-poor nearby WR galaxies (see Kehrig et al. 2013, for more details):

1) The origin of high-ionization nebular lines (e.g. HeII $\lambda 4686$ ), apparently more frequent in high-z galaxies, is still an open question. One widely favored mechanism for $\mathrm{He}^{+}$-ionization involves hot WRs, but it has been shown that nebular HeII $\lambda 4686$ is not 

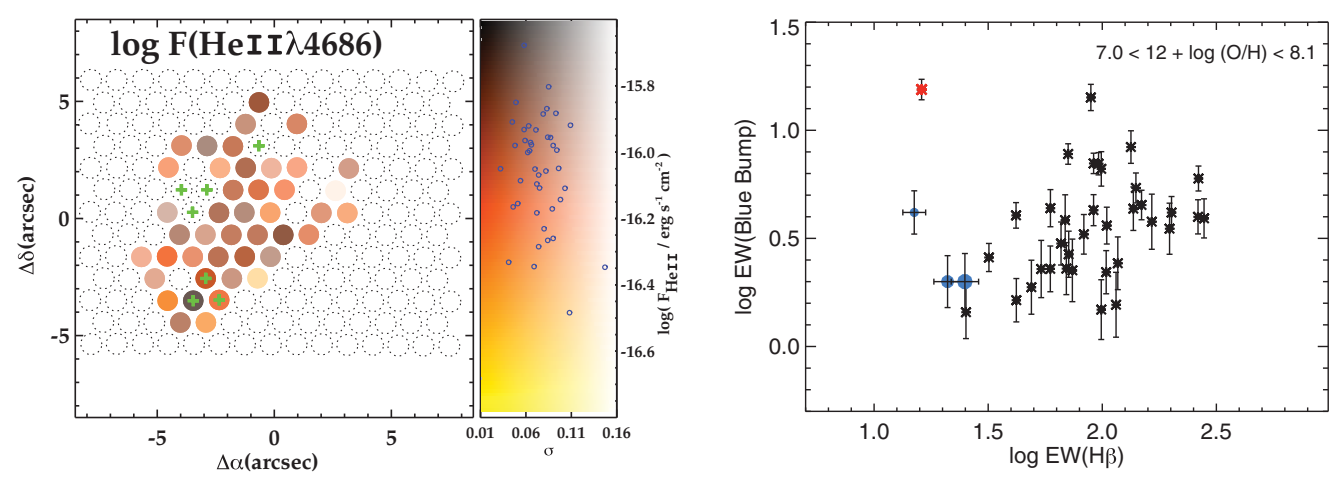

Figure 1. Mrk178 - Left panel: intensity map of the nebular HeII $\lambda 4686$ line; the spaxels where we detect WR features are marked with green crosses. Right panel: EW(WR blue bump) vs $\mathrm{EW}(\mathrm{H} \beta)$. Asterisks show values obtained from SDSS DR7 for metal-poor WR galaxies; the red one represents Mrk178. The three blue circles, from the smallest to the biggest one, represent the 5, 7 and 10 arcsec-diameter apertures from our IFU data centered at the WR knot of Mrk178, at which the SDSS fiber was centered too (Kehrig et al. 2013).

always accompanied by WR signatures, thus WRs do not explain $\mathrm{He}^{+}$-ionization at all times (Kehrig et al. 2008, 2011; Shirazi \& Brinchmann 2012). In Mrk178, we find nebular HeII $\lambda 4686$ emission spatially extended reaching well beyond the location of the WR stars (Fig. 1, left-panel). The excitation source of $\mathrm{He}^{+}$in Mrk178 is still unknown.

2) From the SDSS spectra, we have found a too high EW(WR bump) value for Mrk178, which is the most deviant point among the metal-poor WR galaxies in Fig. 1, right-panel. Using our IFU data, we have demonstrated that this curious behaviour is caused by aperture effects, which actually affect, to some degree, the EW(WR bump) measurements for all galaxies in Fig.1. We have also shown that using too large an aperture, the chance of detecting WR features decreases, and that WR signatures can escape detection depending on the distance of the object and on the aperture size. Therefore, WR galaxy samples/catalogues constructed on single fiber/long-slit spectrum basis may be biased!

\section{Acknowledgements}

This work has been partially funded by research project AYA2010-21887-C04-01 from the Spanish PNAYA.

\section{References}

Cairós L. M., Caon N., Zurita C., et al. 2009, A\& $\&$, 507, 1291

Cairós L. M., Caon N., Zurita C., Kehrig C., Roth M., \& Weilbacher P. 2010, A\&A, 520, A90

Kehrig C., Telles E., \& Cuisinier F. 2004, AJ, 128, 1141

Kehrig C., Vílchez J. M., Telles E., Cuisinier F., \& Pérez-Montero E. 2006, A $\mho A$ A, 457, 477

Kehrig C., Vílchez J. M., Sánchez S. F., et al. 2008, A\&A, 477, 813

Kehrig C., Oey M. S., Crowther P. A., et al. 2011, A\& $A$, 526, A128

Kehrig C., Pérez-Montero E., Vílchez J. M., et al. 2013, MNRAS, 432, 2731

Kunth D. \& Sargent W. L. W. 1981, A\&SA, 101, L5

Leitherer C., et al. 2014, ApJS, 212, id.14

Pérez-Montero E., García-Benito R., Díaz A. I., Pérez E., \& Kehrig, C. 2009, A\&A, 497, 53

Pérez-Montero E.,Kehrig C.,Brinchmann J., et al. 2013, Advances in Astronomy, 2013, id.837392

Shirazi M. \& Brinchmann J. 2012, MNRAS, 421, 1043

Westera P., Cuisinier F., Telles E., \& Kehrig C. 2004, A\&A, 423, 133 